\title{
Staphylococcus lugdunensis: um olhar diferenciado no laboratório clínico
}

Primeira submissão em 29/12/10 Última submissão em 23/02/11 Aceito para publicação em 28/02/11 Publicado em 20/04/11

\author{
Staphylococcus lugdunensis: a different view in the clinical laboratory
}

Alessandro Conrado de Oliveira Silveira'; Pedro Alves d'Azevedo ${ }^{2}$

unitermos
Staphylococcus
lugdunensis
Estafilococos coagulase
negativos
Endocardites
Clumping factor
Cefoxitina

\section{resumo}

Os estafilococos coagulase negativos (ECNs) são cocos Gram-positivos usualmente considerados contaminantes em laboratórios de microbiologia clínica. Apesar de pertencer a este grupo, Staphylococcus lugdunensis pode causar infecções complicadas, como endocardites, infecções de pele e tecidos moles, osteomielites, entre outras. Além da formação de biofilmes, apresenta patogenicidade similar ao Staphylococcus aureus. É um dos principais agentes causadores de endocardites, com taxa de mortalidade de até $70 \%$. Pode ser confundido com $\mathrm{S}$. aureus quando se utilizam testes rápidos para sua identificação, como a pesquisa de clumping factor, no caso de teste de coagulase em lâmina, ou em testes de aglutinação direta em látex. Pode ser facilmente identificado por meio de provas bioquímicas acessíveis, como a presença de atividade da ornitina descarboxilase e pirrolidonil arilamidase (PYR). Apresenta sensibilidade à maioria dos agentes antimicrobianos, devendo ser pesquisada rotineiramente a presença de betalactamases e do gene mecA por meio de testes com cefalosporina cromogênica e suscetibilidade à cefoxitina, respectivamente. Convém salientar que os critérios interpretativos utilizados para avaliar a sensibilidade à cefoxitina são os mesmos preconizados para $S$. aureus e diferentes dos utilizados para os outros ECNs. Apesar de incomum, o S. lugdunensis é um patógeno com acentuada virulência que deve ser corretamente identificado, pois raramente poderá ser considerado contaminante quando isolado de sítios estéreis.

abstract

Coagulase-negatives staphylococci (CNS) are Gram-positives cocci commonly regarded as contaminants in clinical microbiology laboratories. Despite belonging to this group, Staphylococcus lugdunensis may cause complicated infections such as endocarditis, skin infections and soft tissue, osteomyelitis, among others. Apart from the formation of biofilms, it has pathogenic features similar to Staphylococcus aureus. It may be mistakenly identified as S. aureus when using rapid identification tests, such as clumping factor in slide coagulase or in agglutination latex tests. It is easily identified through available biochemical tests, such as the presence of ornithine decarboxylase and pyrrolidonyl arylamidase (PYR). It presents sensitivity to most antimicrobial agents. Furthermore, the presence of beta-lactamase and mecA gene should be routinely investigated by testing with chromogenic cephalosporin and cefoxitin susceptibility, respectively. It is convenient to highlight that the interpretative criteria used to evaluate cefoxitin sensitivity are the same recommended for S. aureus and different from those used for other CNS. Despite the fact it is atypical, S. lugdunensis is a virulent pathogen, which must be accurately identified insofar as it will rarely be deemed as a contaminant when isolated from sterile sites.

\section{key words}

Staphylococcus lugdunensis

Coagulase-negative staphylococci

Endocarditis

Clumping factor

Cefoxitin

1. Mestre em Ciências Farmacêuticas; professor de Microbiologia Clínica e Imunologia Clínica da Fundação Universidade Regional de Blumenau (FURB).

2. Pós-doutor no Laboratório Especial de Microbiologia Clínica (LEMC); professor associado da Universidade Federal de Ciências da Saúde de Porto Alegre (UFCSPA). 


\section{Introdução}

Os estafilococos coagulase negativos (ECNs) são um grupo numeroso e heterogêneo de cocos Gram-positivos, geralmente associados à contaminação de amostras clínicas. Apresentam baixa patogenicidade, necessitando de uma porta de entrada para causar infecções $(7,9,28,30)$.

Entre os ECNs, o Staphylococcus lugdunensis surge como patógeno de características ímpares, pois, apesar de pertencer a este grupo, demonstra patogenicidade semeIhante à do Staphylococcus aureus, apresentando elevadas morbidade e mortalidade associadas ${ }^{(4,11,29)}$.

Atualmente, a prevalência de infecções por S. lugdunensis é baixa, variando de $0,6 \%$ a $13 \%$ entre todos os ECNs isolados em laboratórios de microbiologia clínica. Sua incidência tem aumentado nos últimos anos devido à correta identificação microbiológica ${ }^{(12,14)}$.

Causa essencialmente infeç̧ões em pacientes imunocomprometidos e está associado a extensa gama de processos infecciosos, principalmente infecções de pele e tecidos moles, mas também infecções invasivas, como endocardites, artrite, osteomielite, discite, peritonite, entre

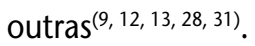

A identificação bioquímica da bactéria apresenta algumas dificuldades. A presença de proteínas de superfície específica pode levar às provas de coagulase em lâmina e pesquisa de clumping factor positivas, sugerindo $S$. aureus. Provas bioquímicas adicionais para confirmação são necessárias, como o teste pirrolidonil arilamidase (PYR) e ornitina descarboxilase ${ }^{(17)}$.

O objetivo dessa revisão é avaliar a identificação de S. lugdunensis, destacar a importância de seu isolamento, descrever sua patogenicidade e discutir os testes de suscetibilidade aos antimicrobianos.

\section{Características}

O S. lugdunensis foi descrito em 1988 por Freney et al..$^{(13)}$. Foi isolado em Lyon, França, explicando a origem do nome, pois lugdunum significa Lyon em latim. É um pequeno coco Gram-positivo (0,8-1 $\mu \mathrm{m}$ de diâmetro), catalase-positivo, podendo ocorrer isolado, aos pares e em cadeias. É um anaeróbio facultativo, podendo crescer em temperaturas de $30^{\circ} \mathrm{C}$ a $45^{\circ} \mathrm{C}$ e em concentrações de cloreto de sódio de até $15 \%{ }^{(11)}$.

Isolados podem apresentar variações na pigmentação e morfologia colonial. As colônias variam de 1 a 4 mm de diâmetro, podendo modificar a coloração para amarelo ou dourado após três a cinco dias de incubação ou permanecer sem pigmentação. A variação na morfologia e pigmentação colonial ocorre devido às pequenas colônias variantes, subpopulações que apresentam crescimento lento, sugerindo a presença de microbiota mista, dificultando a interpretação das culturas ${ }^{(19)}$.

Raramente encontrado na cavidade nasal e isolado mais frequentemente na região perineal, faz parte da microbiota da pele. Em estudo realizado utilizando culturas inguinais de 140 pacientes, houve crescimento de S. lugdunensis em $23 \%$ das mulheres e $19 \%$ dos homens ${ }^{(40)}$.

\section{Discussão}

\section{Patogenicidade}

Como outros ECNs, a habilidade de aderir e formar biofilmes em superfícies estranhas é a principal responsável pela patogenicidade de S. lugdunensis. Os biofilmes são estruturas multicelulares formadas por bactérias fortemente aderidas à superfície e cobertas por uma matriz extracelular polimérica. Promovem proteção contra a resposta imune do hospedeiro, bem como resistência às doses dos antimicrobianos utilizados na terapêutica ${ }^{(10)}$.

O S. lugdunensis se liga a várias proteínas solúveis, como colágeno, imunoglobulina $\mathrm{G}$, fibronectina, plasminogênio, entre outras. Além disso, possui uma proteína que pode se ligar ao fator de von Willebrand. As plaquetas e células endoteliais produzem o fator de von Willebrand, glicoproteína responsável pela ligação de colágeno e plaquetas em locais de injúria vascular ${ }^{(25)}$.

Estudos fornecem evidências que demonstram possuir proteínas de superfície associadas que permitem a adesão bacteriana às células do hospedeiro bem como às superfícies estranhas, um passo crítico na formação de biofilmes. A habilidade de se ligar ao fibrinogênio e ao fator de von Willebrand pode justificar a prevalência do microrganismo em endocardites ${ }^{(12)}$.

O fibrinogênio é a maior proteína presente no plasma sanguíneo, atuando no controle da perda de sangue em tecidos danificados por meio da formação de coágulos de fibrina. Também atua na resposta às infecções, visto que a clivagem enzimática de fibrinogênio em fibrina produz fibrinopeptídeos, que são imunoestimuladores ${ }^{(41)}$.

O S. lugdunensis, assim como o S. aureus, apresenta uma proteína de superfície chamada proteína ligadora de 
fibrinogênio, conhecida também como clumping factor. É codificada por um gene chamado Fbl (S. lugdunensis) e ClfA (S. aureus). É um importante fator de virulência, pois se liga no terminal da cadeia $\gamma$ do fibrinogênio, no mesmo local da integrina plaquetária. As proteínas Fbl e ClfA têm elevada similaridade (62\%), apesar de a proteína do $S$. aureus apresentar uma afinidade 10 vezes maior pelo fibrinogênio. Tal fato pode explicar por que alguns isolados de S. lugdunensis exibem prova rápida para pesquisa de clumping factor negativa. Os anticorpos gerados contra as proteínas Fbl e ClfA podem reconhecer as proteínas heterólogas, sugerindo que apresentem epítopos antigênicos comuns, podendo oferecer proteção cruzada contra S. aureus e S. lugdunensis( ${ }^{(24)}$.

O S. lugdunensis produz uma enzima chamada tanase (tanino acil-hidrolase), capaz de hidrolisar taninos. O gene que codifica para esta proteína é o tanA. Ainda não foram encontrados genes similares no GeneBank. Pode-se utilizar reação em cadeia da polimerase (PCR) para detectar tal gene e confirmar rapidamente uma identificação presuntiva de S. lugdunensis. Ainda não existem métodos padronizados para detecção direta em amostras clínicas, somente para detecção utilizando a colônia bacteriana ${ }^{(26)}$.

O gene regulador acessório (agr) atua como um regulador global dos fatores de virulência, principalmente exoproteínas secretadas, incluindo enterotoxinas, hemolisinas e numerosas enzimas modificadoras de proteínas do hospedeiro. Análise do locus agr de S. lugdunensis mostra uma similaridade de $63 \%$ com S. aureus, explicando a semelhança na patogenicidade entre as duas bactérias ${ }^{(39)}$.

O S. lugdunensis não produz $\alpha, \beta$, ou $\gamma$ hemolisina, mas uma $\delta$-hemolisina, termostável, responsável pela atividade hemolítica. Três pequenos peptídeos são responsáveis por esta função: SLUSH A, SLUSH B e SLUSH C, codificados pelo locus slush (S. lugdunensis sinergistiyc hemolysin) ${ }^{(11)}$.

A lisozima é uma enzima fundamental na resposta imune inata a agentes microbianos. O S. lugdunensis apresenta uma resistência à lisozima de $400 \mathrm{mg} / \mathrm{ml}$. Outras espécies de ECN são suscetíveis a esta enzima. Em S. aureus, a resistência à lisozima é mediada pela presença de uma proteína de membrana (OatA) que acetila o ácido $\mathrm{N}$-acetil murâmico da parede celular, impedindo a ligação da lisozima. O S. lugdunensis possui uma OatA homóloga, indicando que o mecanismo de resistência à lisozima é o mesmo descrito para S. aureus ${ }^{(12)}$.

\section{Aspectos clínicos}

As manifestações clínicas das endocardites causadas por ECN são notadamente diferentes daquelas provocadas por S. aureus. Normalmente, o quadro clínico é sutil e não específico e o curso clínico, mais subagudo ou crônico. Raramente representam ameaça de vida ao paciente, especialmente se tratadas precoce e adequadamente. Entretanto, o S. lugdunensis é uma exceção devido ao seu caráter agressivo ${ }^{(6,8,18,41)}$.

Ao contrário dos outros ECNs, o S. lugdunensis usualmente causa endocardite da válvula nativa, envolvendo principalmente a parte esquerda do coração, frequentemente complicada por uma rápida destruição da válvula (21\%) e formação de abscesso paravalvular (23\%), muitas vezes exigindo a substituição da válvula. Em $32 \%$ dos casos foram verificados embolia sistêmica e focos metastáticos

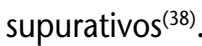

Alguns estudos demonstram a presença de até $18 \%$ de S. lugdunensis entre os ECNs causadores de endocardites, com taxa de mortalidade de $70 \%^{(20,23,32,33,36,38,41)}$.

Vários trabalhos demonstram bacteremia e choque séptico induzidos por S. lugdunensis. Foi relatado um caso que resultou da transfusão de bolsa de plaquetas contaminada. Também há relato de bacteremia e choque séptico 48 horas após extração dentária ${ }^{(12)}$.

Infecções de pele e tecidos moles representam um proeminente número das infecções causadas por $\mathrm{S}$. lugdunensis. Estudo de 63 meses mostrou que 55\% dos 155 isolados de $S$. lugdunensis foram provenientes de abscessos, feridas ou celulites ${ }^{(14)}$.

O S. lugdunensis também é importante causador de infecções ósseas e articulares. Em estudo prospectivo de quatro anos em pacientes submetidos a cirurgias ortopédicas, foi isolado em $3 \%$ do total de $212 \mathrm{ECNs}^{(33)}$. Outro estudo revelou que, durante um período de 40 meses, o $\mathrm{S}$. lugdunensis respondeu por 1\% dos $601 \mathrm{ECNs}$ isolados de pacientes com infecções ortopédicas, incluindo feridas cirúrgicas e próteses infectadas ${ }^{(1)}$. Osteomielite foi documentada em algumas ocasiões. $\mathrm{O}$ microrganismo foi relacionado com osteítes de crânio, infecções posteriores a cirurgias neurológicas, infecção pós-operatória em cirurgias de joeIho, espondilodiscite e osteomielite de vértebra. Trauma e imunossupressão foram fatores de $\operatorname{risco}^{(3,21,22,34,37)}$.

Em estudo com pacientes com e sem infecções orais foi demonstrada a presença de $S$. lugdunensis naqueles com abscessos dentários e osteomielite, porém não foi encontrado em indivíduos saudáveis. Em estudo in vivo, os S. lugdunensis isolados desses pacientes induziram resposta inflamatória e extensas alterações patológicas, como edema intersticial, infiltração inflamatória celular e destruição 
muscular, sugerindo que possa ser o microrganismo associado à doença ${ }^{(42)}$.

Foram relatadas infecções do sistema nervoso central (SNC) relacionadas com o $S$. lugdunensis, incluindo abscessos cerebrais e meningite ${ }^{(16)}$.

Infecções oculares e do trato urinário também foram descritas como causadoras de peritonites ${ }^{(12)}$.

Aspectos laboratoriais

O S. lugdunensis produz acetoína, reduz o nitrato, apresenta atividade de ornitina descarboxilase e PYR, oxidase e fosfatase alcalina negativas, é sensível à novobiocina $\mathrm{e}$ possui variável resistência à polimixina $\mathrm{B}^{(11,12)}$.

Um sinergismo de ação hemolítica de S. lugdunensis, semelhante à atividade das hemolisinas de S. aureus, pode ser demonstrado quando são realizadas estrias perpendiculares de $\beta$-hemolisina estafilocócica (Staphylococcus intermedius) em sangue de carneiro, demonstrada com uma região de completa hemólise na zona de proximidade entre as estrias ${ }^{(11,12) .}$

É um coagulase-negativo, porém uma variável percentual de isolados apresenta clumping factor (coagulase ligada), podendo gerar resultados positivos quando realizado teste rápido de aglutinação em lâmina com plasma ou vários testes comercias de aglutinação em látex ${ }^{(17,43)}$.

A detecção do clumping factoré um teste rotineiramente utilizado para diferenciar $S$. aureus de ECN. Tal teste, realizado sem a coagulase livre (tubo), é a maior causa de erro na identificação de $S$. aureus, visto que o $S$. lugdunensis também pode apresentar clumping factor ${ }^{(4,27)}$.

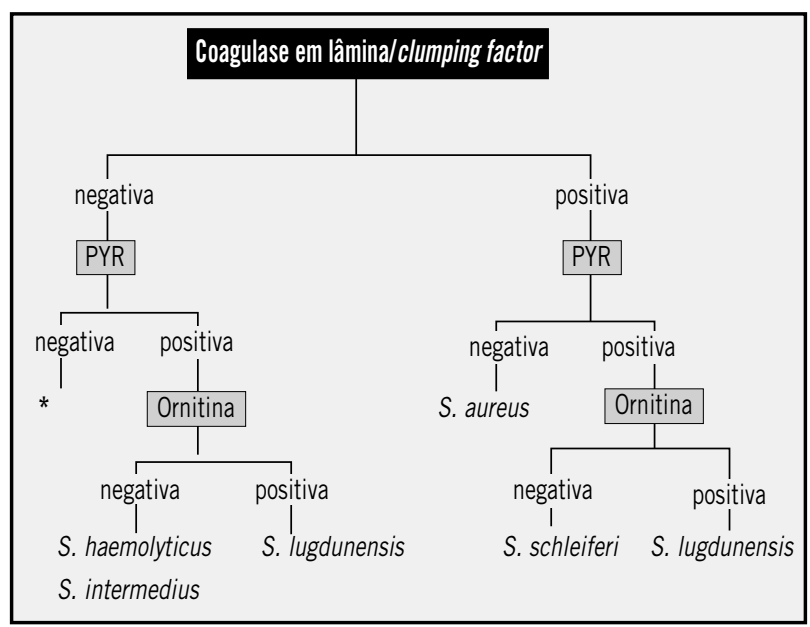

Figura 1 - Fluxograma de identificação simplificada de S. lugdunensis.

Adaptado de POUTANEN, S. M.; BARON, E. J. Staphylococcus lugdunensis: a notably distinct coagulase-negative Staphylococcus. Clinical Microbiology Newsletter, v. 23, n. 19, p. 147-50, 2001.

${ }^{\star} E C N$ s outros que $\mathrm{S}$. lugdunensis, S. haemolyticus, S. schleiferi e S. intermedius. PYR: pirrolidonil arilamidase; ECN: estafilococos coagulase negativo.
Os ECNs não são rotineiramente identificados ao nível de espécie em laboratórios de microbiologia clínica. Normalmente, as culturas com estafilococos são testadas para identificar $S$. aureus, em geral utilizando um teste de aglutinação rápida em lâmina ou pesquisa de proteínas de superfície. Um resultado negativo para o teste em lâmina deve ser seguido de um teste de coagulase em tubo para confirmar tratar-se de um ECN. A intensa virulência associada a S. lugdunensis é uma forte razão para a identificação deste microrganismo ao nível de espécie quando há suspeita de infecção, principalmente se isolada de sítios estéreis ${ }^{(7)}$.

O S. lugdunensis é facilmente identificável com testes bioquímicos acessíveis e rotineiros, como PYR e atividade da ornitina descarboxilase, como demonstrado na Figura $1^{(29)}$.

\section{Testes de suscetibilidade}

O S. lugdunensis, ao contrário dos outros ECNs, permanece sensível à maioria das classes de antimicrobianos. Essa tendência de suscetibilidade, incluindo às penicilinas, não se modifica com base na fonte de infecção. Até hoje foram relatados apenas dois casos de $S$. lugdunensis com presença do gene $\operatorname{mec} A^{(12,15)}$.

Pode ser realizado o teste de pesquisa de betalactamase, utilizando cefalosporina cromogênica (Nitrocefin). Em caso de não produção de betalactamase, o isolado pode ser considerado sensível a todos os betalactâmicos. Caso o teste seja positivo, o isolado é resistente a penicilina, carbóxi, ureído e aminopenicilinas, devendo-se realizar o teste com o disco de cefoxitina para verificar a suscetibilidade aos outros agentes betalactâmicos. A porcentagem de produção de betalactamases em S. Iugdunensis varia de $7 \%$ a $40 \%(2,5,12)$.

Desde 2004, o Clinical Laboratory Standards Institute (CLSI) preconizou o uso do disco de cefoxitina em substituição à oxacilina para predizer a presença do gene mecA. Tal substituição ocorreu por duas razões. Primeiramente, a cefoxitina apresenta melhores sensibilidade e especificidade, principalmente no caso dos ECNs. Segundo, o disco de cefoxitina é de mais fácil leitura que o de oxacilina, cuja leitura deve ser realizada de maneira bastante minuciosa e com luz transmitida. As zonas de inibição formadas ao redor do disco de cefoxitina são claras e podem ser lidas facilmente com luz refletida ${ }^{(5,11,35)}$.

O isolado possuidor do gene mecA produz uma proteína alterada de parede chamada proteína ligadora de penicilina (PBP2a), inviabilizando a terapêutica com qualquer agente betalactâmico. Por isso é fundamental a detecção do gene mecA, seja por características fenotípicas (resistência à 
cefoxitina ou verificação da presença de PBP 2a mediante testes de aglutinação em látex) ou genotípicas (PCR para detecção do gene mecA) $)^{(2,5)}$.

De acordo com o documento M100-S20, os pontos de corte para sensibilidade à cefoxitina são, no teste de discodifusão, halo $\geq 22 \mathrm{~mm}$, e nos testes dilucionais, concentração inibitória mínima $\leq 4 \mu \mathrm{g} / \mathrm{ml}$. No caso de resistência à cefoxitina, o isolado deverá ser considerado resistente a todos os betalactâmicos (penicilinas, cefalosporinas, penicilinas associadas a inibidores de betalactamases e carbapenêmicos), exceto as novas cefalosporinas (ceftobiprol) com atividade anti-MRSA ${ }^{(5)}$.

Cabe salientar a importância da identificação correta do $\mathrm{ECN}$, pois, caso se trate de S. lugdunensis, este apresenta pontos de corte para interpretação do antibiograma iguais aos preconizados para $S$. aureus e diferentes dos utilizados para os outros $\mathrm{ECNs}^{(5)}$.

Em isolados resistentes à eritromicina, deve-se também testar a presença do fenótipo MLSi para detecção da resistência induzível à clindamicina, ensaio conhecido como $D$ teste. No antibiograma, a uma distância de 15 a 26 mm do disco de eritromicina de $15 \mu \mathrm{g}$, deve-se colocar o disco de clindamicina de $2 \mu \mathrm{g}$. Após 16 a 18 horas verifica-se a formação de um achatamento de halo próximo ao disco de clindamicina, conforme demonstra a Figura 2. O isolado deverá ser reportado como resistente à clindamicina ${ }^{(5)}$.

Um fenômeno interessante que ocorre com S. lugdunensis é a tolerância à vancomicina. Em isolados sensíveis à vancomicina, são refratários em ensaios de atividade bactericida, que podem ser associados a uma razão da concentração bactericida mínima (CBM) e concentração inibitória

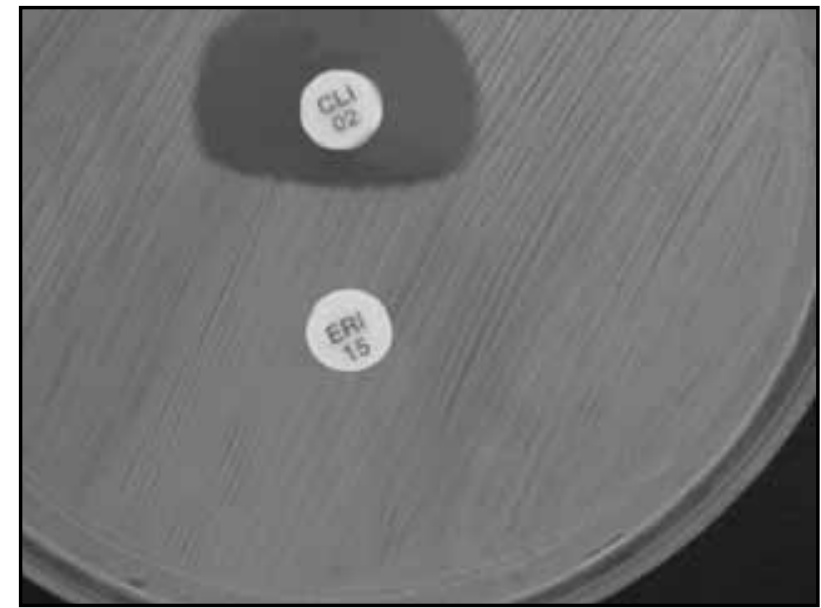

Figura 2 - D teste: achatamento de halo de clindamicina demonstrando resistência induzível

Fonte: Caio Roberto Salvino.

mínima $(\mathrm{CIM}) \geq 32 \mu \mathrm{g} / \mathrm{ml}$. Noventa e três por cento de S. lugdunensis sensíveis à vancomicina (CIM de 0,5 a $2 \mu \mathrm{g} / \mathrm{ml}$ ) demonstraram CBM $\geq 128 \mu \mathrm{g} / \mathrm{ml}$. Um segundo estudo demonstra resultados similares para teicoplanina. Outros estudos tornam-se necessários para determinar a relevância clínica desses achados ${ }^{(12)}$.

\section{Conclusão}

O S. lugdunensis é um patógeno emergente que tem assumido importância nos últimos 20 anos devido à sua elevada virulência. É fundamental a sua correta identificação, pois raramente poderá ser considerado um contaminante quando isolado de fluidos corporais estéreis.

\section{Referências}

1. ARCIOLA, C. R. et al. Prevalence and antibiotic resistance of 15 minor staphylococcal species colonizing orthopedic implants. The International Journal of Artificial Organs, v. 29, p. 395-401, 2006.

2. BATISTA, N. et al. Evaluación de métodos para el estudio de la sensibilidad a la oxacilina y penicilina em 60 aislamientos de Staphylococcus Iugdunensis. Enfermedades Infecciosas y Microbiologia Clínica, v. 27, n. 3, p. 148-52, 2009.

3. CAMACHO, M. et al. Three-year outcome in a patient with Staphylococcus lugdunensis discitis. Joint Bone Spine, v. 69, p. 85-7, 2002.

4. CERCENADO, E. Staphylococcus lugdunensis: un estafilococo coagulasa negativo diferente de los demás. Enfermedades Infecciosas y Microbiología Clínica, v. 27, n. 3, p. 139-42, 2009.

5. CLINICAL LABORATORY STANDARDS INSTITUTE. Performance Standards for Antimicrobial Susceptibility Testing; Twentieth Informational Supplement. CLSI document M100-S20. Clinical and Laboratory Standards Institute, Wayne, Pennsylvania, 2010.

6. CHOPRA, A. et al. Intracardiac lead endocarditis due to Staphylococcus Iugdunensis. International Journal of Infectious Diseases, IN PRESS, 2010.

7. CROSSLEY, K. B. et al. Staphylococci in human disease. 2. ed. Wiley-Blackwell; 2009.

8. EBRIGHT, J. R.; PENUGONDA, N.; BOWN, W. Clinical experience with Staphylococcus lugdunensis bacteremia: a retrospective analysis. Diagnostic Microbiology and Infectious Disease, v. 48, p. 17-21, 2004.

9. EIFF, C. V.; PETERS, G.; HEILMANN, C. Coagulase-negative staphylococci as nosocomial pathogens. The Lancet Infectious Diseases, v. 2, p. 677-85, 2002.

10. FRANK, K. L.; PATEL, R. Intravenously administered pharmaceuticals impact biofilm formation and detachment of Staphylococcus lugdunensis and other staphylococci. Diagnostic Microbiology and Infectious Disease, v. 60, p. 9-16, 2008. 
11. FRANK, K. L.; PATEL, R. Staphylococcus lugdunensis: not the average coagulase-negative Staphylococcus species. Clinical Microbiology Newsletter, v. 30, n. 8, p. 55-62, 2008.

12. FRANK, K. L.; POZO, J. L.; PATEL, R. From clinical microbiology to infection pathogenesis: how daring to be different works for Staphylococcus lugdunensis. Clinical Microbiology Reviews, v. 21, n. 1, p. 111-33, 2008.

13. FRENEY, J. et al. Staphylococcus lugdunensis sp. nov. and Staphylococcus schleiferi sp. nov., two species from human clinical specimens. International Journal of Systematic Bacteriology, v. 38, p. 168-72, 1988.

14. HERCHLINE, T. E.; AYERS, L. W. Occurrence of Staphylococcus lugdunensis in consecutive clinical cultures and relationship of isolation to infection. Journal of Clinical Microbiology, v. 29, n. 3, p. 419-21, 1991.

15. HERCHLINE, T. E. et al. Penicillinase production and in vitro susceptibilities of Staphylococcus lugdunensis. Antimicrobial Agents and Chemotherapy, v. 34, n. 12, p. 2434-5, 1990.

16. HONG, T.; SPERBER, S. J.; TANG, Y. W. Recurrent Staphylococcus lugdunensis central nervous system infection associated with a ventricular peritoneal shunt. Antimicrobics and Infectious Diseases Newsletter, v. 18, n. 12, p. 91-3, 2002.

17. IEVEN, M. et al. Rapid and economical method for species identification of clinically significant coagulase-negative staphylococci. Journal of Clinical Microbiology, v. 33, n. 5, p. 1060-3, 1995.

18. KOH, T. W.; BRECKER, S. J. D.; LAYTON, C. A. Successful treatment of Staphylococcus lugdunensis endocarditis complicated by multiple emboli: a case report and review of the literature. International Journal of Cardiology, v. 55, p. 193-7, 1996.

19 LEUNG, M. J. et al. Colony variation in Staphylococcus Iugdunensis. Journal of Clinical Microbiology, v. 36, n. 10, p. 3096-8, 1998.

20. MATTHEWS, P. C. et al. Staphylococcus lugdunensis endocarditis following cardiac catheterization. International Journal of Cardiology, v. 130, p. 87-8, 2007.

21 MUÑOZ, M. L. et al. Artritis séptica por Staphylococcus lugdunensis. Reumatología Clínica, v. 5, n. 1, p. 44-5, 2009.

22. MURDOCH, D. R. et al. Vertebral osteomyelitis due to Staphylococcus lugdunensis. Journal of Clinical Microbiology, v. 34, n. 4, p. 993-4, 1996.

23. MURILLO, J.; McMAHON, C.; STARR, J. Large vegetations in Staphylococcus lugdunensis endocarditis. Heart \& Lung, v. 37, n. 6, p. 479-80, 2008.

24. NILSSON, M.; BJEKETORP, F.; GUUS, B.; FRYKBERG, L. A fibrinogen-binding protein of Staphylococcus /ugdunensis. FEMS Microbiology Letters, v. 241, p. 87-93, 2004.

25. NILSSON, M. et al. A von Willebrand factor-binding protein from Staphylococcus lugdunensis. FEMS Microbiology Letters, v. 234, p. 155-61, 2004.

26. NOGUCHI, N. et al. Using the tannase gene to rapidly and simply identify Staphylococcus Iugdunensis. Diagnostic Microbiology and Infectious Disease, v. 66, p. 120-3, 2010.

27. PERSONNE, P. et al. Comparative performances of six agglutination kits assessed by using typical and atypical strains of Staphylococcus aureus. Journal of Clinical Microbiology, v. 35, n. 5, p. 1138-40, 1997.

28. PIETTE, A.; VERSCHRAEGEN, G. Role of coagulasenegative staphylococci in human disease. Veterinary Microbiology, v. 134, p. 45-54, 2009.
29. POUTANEN, S. M.; BARON, E. J. Staphylococcus lugdunensis: a notably distinct coagulase-negative Staphylococcus. Clinical Microbiology Newsletter, v. 23, n. 19, p. 147-50, 2001.

30. ROGERS, K. L.; FEY, P. D.; RUPP, M. E. Coagulase-negative staphylococcal infections. Infectious Disease Clinics of North America, v. 23, p. 73-98, 2009.

31. SCHNITZLER, N. et al. Staphylococcus lugdunensis: report of a case of peritonitis and an easy-to-perform screening strategy. Journal of Clinical Microbiology, v. 36, n. 3, p. 812-3, 1998.

32. SEENIVASAN, M. H.; YU, V. L. Staphylococcus lugdunensis endocarditis: the hidden peril of coagulase-negative staphylococcus in blood cultures. European Journal of Clinical Microbiology and Infectious Diseases, v. 22, p. 489-91, 2003.

33. SHUITLEWORTH, R.; COLBY, W. D. Staphylococcus Iugdunensis endocarditis. Journal of Clinical Microbiology, v. 30, n. 8, p. 1948-52, 1992.

34. SIVADON, V. et al. Use of genotypic identification by sodA sequencing in a prospective study to examine the distribution of coagulase-negative Staphylococcus species among strains recovered during septic orthopedic surgery and evaluate their significance. Journal of Clinical Microbiology, v. 43, p. 2952-4, 2005.

35. SWENSON, J. M.; SKOV, R.; PATEL, J. B. The cefoxitin disk test: what a clinical microbiologist needs to know. Clinical Microbiology Newsletter, v. 29, n. 5, p. 33-40, 2007.

36. TAKAHASHI, N. et al. The pitfall of coagulase-negative Staphylococci: a case of Staphylococcus lugdunensis endocarditis. International Journal of Cardiology, v. 137, p. 15-7, 2009.

37. THOMAS, S.; HOY, C.; CAPPER, R. Osteomyelitis of the ear canal caused by Staphylococcus Iugdunensis. Journal of Infection, v. 53, 227-9, 2006.

38. VANDENESCH, F. et al. Endocarditis due to Staphylococcus Iugdunensis: report of 11 cases and review. Clinical Infectious Diseases, v. 17, p. 871-6, 1993.

39. VANDESNESCH, F. et al. Agr-related sequences in Staphylococcus lugdunensis. FEMS Microbiology Letters, v. 111, p. 115-22, 1993.

40. VAN DER MEE-MARQUET, N. et al. Staphylococcus lugdunensis infections: high frequency of inguinal area carriage. Journal of Clinical Microbiology, v. 41, p. 1404-9, 2003.

41. VIGANEGO, F. et al. Staphylococcus lugdunensis endocarditis following lower extremity revascularization. International Journal of Cardiology, v. 116, p. 4-6, 2007.

42. YOU, Y. O. et al. Staphylococcus lugdunensis: a potential pathogen in oral infection. Oral Surgery Oral Medicine Oral Pathology, v. 88, n. 3, p. 297-302, 1999.

43. ZBINDEN, R. et al. Detection of clumping factor-positive Staphylococcus lugdunensis by Staphaurex Plus ${ }^{\circledR}$. Journal of Microbiological Methods, v. 31, p. 95-8, 1997.

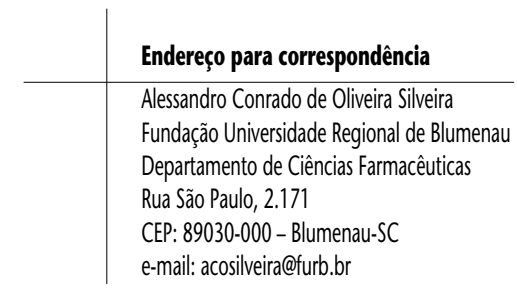

